\title{
CORRECTION
}

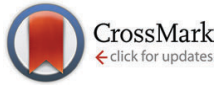

Cite this: J. Mater. Chem. C, 2016, 4, 1126

DOI: $10.1039 / \mathrm{cttc} 90020 \mathrm{~h}$

www.rsc.org/MaterialsC

\section{Correction: Highly efficient and stable blue polymer light emitting diodes based on polysilafluorenes with pendent hole transporting groups}

\author{
Guangrong Jin, ${ }^{a}$ Lianpeng Xia, ${ }^{\mathrm{b}}$ Zhe Liu, ${ }^{\mathrm{b}}$ Hong Lin, ${ }^{\mathrm{a}}$ Jun Ling, ${ }^{\mathrm{c}}$ Hongbin Wu, ${ }^{\mathrm{a}}$ \\ Lintao Hou*b and Yueqi Mo*a
}

Correction for 'Highly efficient and stable blue polymer light emitting diodes based on polysilafluorenes with pendent hole transporting groups' by Guangrong Jin et al., J. Mater. Chem. C, 2016, DOI: 10.1039/ c5tc03665h.

The first funding number quoted for the Natural Science Foundation of China in the acknowledgments is incorrect and the funding number 21474129 should be replaced with 21174042. The correct version of the acknowledgements is given below.

The Royal Society of Chemistry apologises for these errors and any consequent inconvenience to authors and readers.

\section{Acknowledgements}

This work was financially supported by Natural Science Foundation of China (21174042, 11204106, and 61274062), Fund of Project on the Integration of Industry, Education and Research of Guangdong Province (2013B090500072), the Open Fund of the State Key Laboratory of Luminescent Materials and Devices (South China University of Technology 2012-skllmd-10) and the Fundamental Research Funds for the Central Universities. Thanks to Dr X. W. Chen for his work on the Yamamoto polymerization and to Miss Tan Qiong-Xuan for the artwork of the front cover.

\footnotetext{
${ }^{a}$ College of Materials Science and Engineering, South China University of Technology, Guangzhou 510640, China. E-mail: pomoy@scut.edu.cn

${ }^{b}$ Siyuan Laboratory, Department of Physics, Jinan University, Guangzhou 510632, China. E-mail: thlt@jnu.edu.cn

${ }^{c}$ MOE Key Laboratory of Macromolecular Synthesis and Functionalization, Department of Polymer Science and Engineering, Zhejiang University, Hangzhou 310027, China
} 